Editor's Note: These short, critical reviews of recent papers in the Journal, written exclusively by graduate students or postdoctoral fellows, are intended to summarize the important findings of the paper and provide additional insight and commentary. For more information on the format and purpose of the Journal Club, please see http://www.jneurosci.org/misc/ifa_features.shtml.

\title{
What's the Buzz about Honeybee Memory?
}

\author{
Scott E. Dobrin ${ }^{1}$ and Daniel E. Wilson ${ }^{2,3}$ \\ ${ }^{1}$ Neuroscience Program, Wake Forest University Graduate School of Arts and Sciences, Winston-Salem, North Carolina 27157, ${ }^{2}$ Duke University, Durham, \\ North Carolina 27705, and ${ }^{3}$ Department of Biology, Wake Forest University, Winston-Salem, North Carolina 27157 \\ Review of Müßig et al.
}

Although glutamate is known to be critical to vertebrate neuronal plasticity, its function in the insect brain is poorly understood and the NMDA receptor (NMDAR) has only recently been investigated in the honeybee. NMDARs are ligand-gated, voltagesensitive cation channels composed of NR1 and NR2 subunits (Zannat et al. 2006). The NR1 subunit, which is critical for functional expression of NMDARs, is expressed throughout the honeybee brain (Zannat et al., 2006), and pharmacological blockade of these receptors impairs memory formation (Si et al., 2004). In a recent report by Müßig et al. (2010) in The Journal of Neuroscience, the effects of disrupting the NMDARs through RNA interference (RNAi) was examined in the honeybee.

The proboscis extension response is a common appetitive olfactory conditioning task used in insects. Honeybees (and other insects, including fruit flies) reflexively extend their proboscis, an insect mouthpart, when sucrose [the unconditioned stimulus (US)] is touched to the antenna. If regularly paired with a specific odor [the conditioned stimulus (CS)], a bee quickly learns the association and extends its proboscis when presented with the odor alone (for review, see Fahrbach and Dobrin, 2009). This

Received July 29, 2010; revised Sept. 3, 2010; accepted Sept. 8, 2010.

This work was supported by the National Institutes of Health (Grant R01 GM073644A) and Wake Forest University.

We thank Susan Fahrbach for her insightful comments on this manuscript.

Correspondence should be addressed to Scott E. Dobrin, Wake Forest University, 1834 Wake Forest Road, Winston Hall, Biology Department, Winston-Salem, NC 27106. E-mail: sdobrin@wfubmc.edu.

DOI:10.1523/JNEUROSCI.3951-10.2010

Copyright $\odot 2010$ the authors $\quad 0270-6474 / 10 / 3014593-02 \$ 15.00 / 0$ basic paradigm is robust: a stable longterm memory forms in as few as three CS-US pairings, as in Müßig et al. (2010). The antennal lobes (ALs), the primary olfactory neuropils, and the mushroom bodies (MBs), structures that receive inputs from all primary sensory neuropils, have been identified as sites of convergence of the CS-US signals in the insect brain.

The experiments performed by Müßig et al. (2010) represent one of the few published examples of successful in vivo knock down of a gene within the honeybee brain. Previously, investigators have used antisense to knock down protein kinase A (Fiala et al., 1999), and RNAi against a receptor for the insect biogenic amine octopamine (Farooqui et al., 2003) to investigate the mechanisms of memory. In the present study, both double-stranded RNA (dsRNA) and small-interfering RNAs were used to knock down the NR1 subunit in honeybee MBs. The use of two RNAi approaches, appropriate negative controls, and antibodies to two different epitopes makes these results compelling. One day following dsNR1 injection, NR1 protein was reduced by 30\% [Müßig et al. (2010), their Fig. 2], returning to baseline by the second day. Although a more sensitive measure of the effectiveness of the NR1 knockdown might have been obtained by directly measuring NR1 transcript abundance through quantitative real-time PCR, the present study is strong because ultimately it is changes in protein expression that directly influence behavior.

Short-term memory (STM), mediumterm memory (MTM), and long-term memory (LTM) are regarded as temporally and mechanistically distinct phases of memory. The molecular mechanisms of the each memory phase are still being investigated, but mounting evidence indicates each is triggered by the same event but processed independently (for review, see Schwärzel and Müller, 2006; Menzel, 2009). STM, stored for seconds, was not examined in this article. MTM forms following three conditioning trials and lasts hours. It requires the calcium-dependent protease calpain to cleave PKC, creating the constitutively active isoform PKM. Calpain inhibition in the AL results in a loss of only MTM. LTM, which can be subdivided into early (eLTM) and late (ILTM) phases, occurs on the order of days. eLTM (memories lasting 1-2 d) requires translation of new proteins, whereas ILTM (memories lasting multiple days to weeks) depends on both transcription and translation. The distinct yet related mechanisms of eLTM and ILTM are better understood. Both require activation of PKA by cAMP, as blocking the activity of either inhibits all LTM. LTM formation also requires nitric oxide. Nitric oxide putatively targets cGMP, which directly prolongs the activation of PKA, a critical step in LTM formation. While PKA is involved in both LTM phases, experimental manipulations indicate distinct pathways. Bees fed before olfactory conditioning have low basal PKA activity levels and do not perform well in the learning task. Experimentally elevating PKA rescues ILTM but not STM, MTM, or eLTM. Local protein translation using preexisting mRNA occurs during the forma- 
tion of eLTM, whereas ILTM requires transcription and translation of new proteins.

Because of the known role of NMDARs in memory formation in vertebrates and the requirement of the NR1 subunit for functional NMDARs, the authors tested how NR1 reduction affected learning using the proboscis extension response. Bees with dsNR1 injected directly into the brain performed more poorly during the acquisition phase of memory as well as the tests of MTM and eLTM when compared with control bees [Müßig et al. (2010), their Fig. 4]. One day following injection of dsRNA, bees were olfactory conditioned with three sucroseodor pairings. The odor was then presented alone $2 \mathrm{~h}, 2 \mathrm{~d}$, or $3 \mathrm{~d}$ after training to test MTM, eLTM, or ILTM, respectively. The percentage of bees that extend their proboscis during the odor presentation but before the sucrose administration during training was used as a measure of acquisition. Differences between dsNR1 and control bees were seen by the second pairing [Müßig et al. (2010), Fig. 4A]. This suggests that efficient acquisition requires functional NMDARs. During the memory retrieval tests, only MTM and eLTM, not ILTM, were affected by NR1 reduction [Müßig et al. (2010), their Fig. 4A]. The effects on memory retrieval may reflect a deficit in acquisition. To control for this, the authors reanalyzed the data using only those bees that responded to the odor in the third CS-US pairing [Müßig et al. (2010), their Fig. 5]. These bees still showed a deficit in MTM. We would appreciate a similar reanalysis of the eLTM data as well.

To dissect the effect of NMDAR function on memory recall, the authors also asked whether dsRNA injection after training affects olfactory learning. Bees were trained as in the initial study, injected with dsNR1 2 days later, and tested an additional day later. This design tests the effects of NR1 reduction on ILTM only (testing $3 \mathrm{~d}$ after training). The authors found no difference between dsNR1 and control injected bees [Müßig et al. (2010), their Fig. 7] as observed in the previous experiments. It is possible that injecting dsNR1 immediately after training (or with a smaller delay) would allow confirmation of reductions in MTM or eLTM, both of which were affected in the preinjection test.

The role of glutamate in forming olfactory memories in the honeybee is unclear.
Photolytic uncaging of glutamate in MB immediately following a single sucrose-odor pairing resulted in improved response to the odor alone $48 \mathrm{~h}$ later (eLTM) (Locatelli et al., 2005). The finding by Müßig et al. (2010) that disruption of NMDAR function affects memory formation potentially offers a more precise mechanism for the role of glutamate in learning. Interestingly, when the investigators measured NR1 protein levels in the dorsal region of the central brain via Western blot $2 \mathrm{~h}$ following training no difference was found between the RNAi and control bees [Müßig et al. (2010), their Fig. $3 B]$. This suggests that conditioning invoked an upregulation of NMDARs. Although it is possible that de novo protein expression can be completed in that time frame, it is most likely that synaptic mRNA, which was not degraded from RNAi treatment, was translated. This may represent a biologically relevant increase in functional NMDARs in the membrane, which would influence calcium-dependent processes, or an overwhelming of the RNAi effect, as the authors suggest. A follow-up experiment using calcium imaging together with pharmacological manipulations of the brains of bees previously conditioned would help answer this question.

A recent report documented an anatomical substrate of a memory trace in the honeybee (Hourcade et al., 2010). A higher density of microglomeruli (synaptic specializations) in the olfactory, but not visual, region of the $\mathrm{MB}$ was found in conditioned bees compared with control bees. An increased density of microglomeruli may reflect synaptogenesis. It would be interesting to examine structural plasticity of the microglomeruli following olfactory training in bees with NR1 reduction. We hypothesize that the memory deficits induced by blocking NMDAR function would be reflected by no change in the density of microglomeruli in the olfactory region of the mushroom body. This would suggest an NMDARmediated mechanism for inducing structural plasticity via olfactory learning and provide new details of molecular and cellular memory processing.

In summary, Mü $\beta$ ig and colleagues provide a clear demonstration that RNA interference in the honeybee brain is effective and reversible in vivo, although the treated bees are harnessed in the laboratory rather than free-flying in the field. Furthermore, by inhibiting NMDAR function the involvement of glutamate signaling in honeybee olfactory learning has been better understood; functional NMDARs are necessary for the formation of MTM and eLTM, but not ILTM. These findings support the hypothesis that parallel but distinct cascades are involved in the formation of the different memory phases. We predict that future studies combining injections of dsRNA with behavioral, electrophysiological, and neuroanatomical methods will yield exciting new insights into our understanding of the molecular mechanisms of memory.

\section{References}

Fahrbach SE, Dobrin SE (2009) The how and why of structural plasticity in the adult honey bee brain. In: Cognitive ecology II (Dukas R, Ratcliffe JM, eds), pp 37-48. Chicago: University of Chicago Press.

Farooqui T, Robinson K, Vaessin H, Smith BH (2003) Modulation of early olfactory processing by an octopaminergic reinforcement pathway in the honeybee. J Neurosci 23:5370-5380.

Fiala A, Müller U, Menzel R (1999) Reversible downregulation of protein kinase A during olfactory learning using antisense technique impairs long-term memory formation in the honeybee, Apis mellifera. J Neurosci 19:10125-10134.

Hourcade B, Muenz TS, Sandoz JC, Rössler W, Devaud JM (2010) Long-term memory leads to synaptic reorganization in the mushroom bodies: a memory trace in the insect brain? J Neurosci 30:6461-6465.

Locatelli F, Bundrock G, Müller U (2005) Focal and temporal release of glutamate in the mushroom bodies improves olfactory memory in Apis mellifera. J Neurosci 25:11614-11618.

Menzel R (2009) Conditioning: simple neural circuits in the honeybee. In: Encyclopedia of neuroscience (Squire L, ed). New York: Academic.

Müßig L, Richlitzki A, Rößler R, Eisenhardt D, Menzel R, Leboulle G (2010) Acute disruption of the NMDA receptor subunit NR1 in the honeybee brain selectively impairs memory formation. J Neurosci 30:7817-7825.

Schwärzel M, Müller U (2006) Dynamic memory networks: dissecting molecular mechanisms underlying associative memory in the temporal domain. Cell Mol Life Sci 63:989-998.

Si A, Helliwell P, Maleszka R (2004) Effects of NMDA receptor antagonists on olfactory learning and memory in the honeybee (Apis mellifera). Pharmacol Biochem Behav 77:191-197.

Zannat MT, Locatelli F, Rybak J, Menzel R, Leboulle $\mathrm{G}$ (2006) Identification and localisation of the NR1 sub-unit homologue of the NMDA glutamate receptor in the honeybee brain. Neurosci Lett 398:274-279. 